\title{
Research on 50 Clinical Nursing Cases in Gynecologic Malignant Tumors Treatment
}

\author{
Fuling Fan $^{1, a}$ \\ ${ }^{1}$ Luohe Medical College, Luohe, Henan, China, 462000 \\ aemail,
}

Keywords: Psychological Intervention; Nursing; Malignant; Gynecological

\begin{abstract}
The object is to explore the psychological intervention and nursing method in gynecologic malignancies during chemotherapy and improve clinical care effect. The methods since March 2012 - May 2014 were treated: 50 cases of gynecologic cancer patients were randomly divided into two groups (psychological intervention group) and control group (usual care group) 80 cases and two groups were compared after chemotherapy mental state and nursing satisfaction with the situation. Results observation group and control group similar interventions SDS score, and SDS scores after the intervention in the observation group than in the control group were significantly different, and the degree of satisfaction higher in the control group $(\mathrm{P}<0.01)$, with statistical significance. During the implementation of the conclusions of gynecological cancer chemotherapy nursing intervention can significantly improve the patient's mental state, improve nursing satisfaction, improving the quality of life of patients is important.
\end{abstract}

\section{Introduction}

Gynecologic malignancies in possession of a significant proportion of tumor diseases, gynecological malignancies accounting for $12 \%$ of all female cancer to $15 \%$, which is a serious threat to women's health and life and fertility diseases, of which about $21 \%$ of malignant tumors No fertility in young women. Systemic chemotherapy is an important method of treatment of gynecologic malignancies, which can improve the quality of life of patients and prolong survival, but long courses of chemotherapy, adverse reactions during treatment, patients with poor tolerance features, making cancer patients during chemotherapy nursing intervention is crucial. Good psychological intervention gynecologic malignancies during chemotherapy and other treatments can improve patient tolerance to chemotherapy to reduce adverse events and improve the quality of life of patients. In this paper, our hospital from 160 cases in recent years of psychological intervention during chemotherapy in patients with gynecologic malignancies and other care to make the relevant set forth in order to help clinical care, as follows.

\section{Materials and Methods}

General Information. Our hospital were randomly divided into two groups (psychological intervention group) and control group (usual care group) 25 cases since March 2012 - May 2014 were treated 50 cases of gynecological cancer patients, all patients were diagnosed as malignant gynecological Cancer patients and exclude unstable condition, mental illness, accompanied by disturbance of consciousness. Observation group of 25 patients aged 21 to 76 years, mean (46.8 \pm 3.2) years; tumor types: nine cases of cervical cancer, ovarian cancer, 7 cases of endometrial carcinoma in 6 cases, the other three cases; 1 to 5 times the number of chemotherapy average (2.6 \pm 0.3) times; cultural level: primary school education in 4 cases, 7 cases of junior high school, high school and higher education in 14 cases. The control group of 25 patients aged 23 to 78 years, mean (47.2 \pm 3.4$)$ years; tumor types: 10 cases of cervical cancer, ovarian cancer seven cases, five cases of endometrial cancer, the other three cases; 1-6 times the number of chemotherapy average (2.8 \pm 0.4) times; cultural level: primary school education in 5 cases, 9 cases of junior high school, high school and higher education in 11 cases. Comparison of the two groups in terms of age, education level, type of disease and other areas of little difference ( $\mathrm{P}>0.05)$, comparable. Chemotherapy both 
groups of patients had PAC (cisplatin, doxorubicin, cyclophosphamide), PC (cisplatin, cyclophosphamide), PEB (cisplatin, VP16, PYM), PVB (cisplatin, vincristine base, BLM), VAC (vincristine, dactinomycin, cyclophosphamide) and the like.

Nursing Method. Psychological Intervention cancer Once diagnosed, patients often wonder, anxiety, sadness, depression, despair and other complex emotions, and these negative emotions tend to affect confidence in the treatment and the implementation process, improve mental and emotional patient during treatment, can enhance the confidence of patients and medical staff to actively cooperate with the work to improve the treatment effect is very important. Care workers should be sympathetic, patience and careful patient effective psychological counseling, given the appropriate psychological intervention for patients with different mental states. Correctly guide patients to recognize the cancer is not incurable and cancer is also a special "chronic." Introduces chemotherapy to patients and can be achieved, and describes cases after chemotherapy achieved a significant effect, so that the patient hopes confidence aggressive treatment. Managed to get close with the families of patients, relatives and units, try to solve the difficulties of diseases other than set the public whereas repeated enlighten comfort, caring, patient, spend more time with patients, encourage patients to feel good, doing outdoor activities, fresh air, and accompany patients qigong, tai chi and other movement. Enable patients to fully experience the beauty of life, feel the warmth of the body in the family, recognizing the value of your life, to re-establish a correct outlook on life and values, brave suffering from the disease, in order to adhere to chemotherapy and lay a good psychological foundation.

Gastrointestinal reactions care most anticancer drugs are gastrointestinal mucosa. There is some damage. During chemotherapy patients often have loss of appetite, nausea, vomiting and other gastrointestinal symptoms. Because chemotherapy for the body's energy consumption is huge, with patients with gastrointestinal discomfort prone to anorexia, seriously affected the intake and the body's absorption of nutrients, so that more patients with low resistance, resistance to chemotherapy in serious decline. Care workers should strengthen patient care diet, try to pay attention to in the diet and other aspects of the deployment of color, flavor, taste and strive for patients with diet, dietary changes and types of flavors to smaller meals for the diet principles; be patient eat some of the spleen vomiting food, such as ginger, orange peel, plum, etc; ensure clean patient comfortable dining environment, suitable dining place should be ventilated to prevent odors affect the patient appetite; meal when the patient may play for patients like to hear music, or for patients about some inconsequential topic, let the patient distract the attention of gastrointestinal reactions can be more into the diet; patients after a meal should rest for $1 \mathrm{~h}$, after some appropriate guide their activities, can promote diet digest absorption; to guide patients to maintain good oral hygiene habits, require patients to get up early, sleep, have to mouthwash after meals, can effectively reduce the symptoms of anorexia because of nausea and vomiting caused. In patients feel nausea, vomiting patient to relax muscles feeling row slow, deep breaths can reduce nausea and vomiting.

Myelosuppression care to keep the environment clean, establish a strict disinfection and isolation system; during chemotherapy should pay strict attention to changes in blood of patients; administered to the patient increase in protein, vitamins, food intake, to enhance the body's resistance, but should pay attention to food hygiene, avoid raw vegetables and fruit. When taking invasive procedures, we should strictly abide by the principles of aseptic technique to avoid iatrogenic infection. Examination showed that platelet decline, should closely observe the changes in complexion and vital signs of patients, early detection of bleeding caused by thrombocytopenia. Appropriate restrictions according to the disease activity in patients, due to frail weakness, anemia and bleeding caused by trauma accident prevention activities when, in patients with intracranial hemorrhage or other internal bleeding tendency should be absolute bed rest. Patient is asked to get rid of bad habits, such as pull the nose, biting, etc., to prevent bleeding and infection.

Evaluation. The two groups of patients were treated with self-rating depression scale (SDS) before and after chemotherapy mental health assessment and questionnaire form patient care satisfaction survey.

Statistical Methods. The data set using SPSS15.0 statistical software for processing 
measurement data using $\mathrm{x} \pm \mathrm{s}$ that the groups t test, measurement data using the chi-square test, are $\mathrm{P}<0.05$ was considered statistically significant.

\section{Results}

Observation group and control group before the nursing intervention, the SDS scores similar, the difference was not statistically significant. And after psychological care, nursing care of complications after the observation group SDS score was significantly reduced compared with the control group, the difference was significant, statistical analysis, $\mathrm{P}<0.01$. And after my hospital comprehensive clinical nursing intervention, due to give a patient a comprehensive, integrated care, patient care more satisfied, there is a significant difference compared with the control group comparison, $\mathrm{P}<0.01$, the difference was statistically significant.

\section{Discussion}

Chemotherapy is one of the important measures for the treatment of gynecologic malignancies, since cancer patients often lack sufficient knowledge during chemotherapy prone to a variety of adverse psychological state, and produce these adverse psychological states will directly affect the confidence of patients and to cooperate with health care work. A long period of instability and negative psychological state, it will seriously affect the quality of life of patients and greatly reduce the effectiveness of chemotherapy. During chemotherapy for patients with gynecologic malignancies preventive care necessary psychological care and intervention-related adverse reactions, can greatly improve patient tolerance to chemotherapy, chemotherapy and actively cooperate with treatment, and reduce the possibility of adverse reactions during treatment. Based on the observation group care for patients with psychological intervention, a common complication of pre

See nursing, the results of statistics showed that the observation group after nursing intervention, SDS score was significantly reduced compared with the control group, the difference was significant, statistical analysis, $\mathrm{P}<0.05$. In addition, I also summed up the hospital patient satisfaction with nurses observe patients for nurses care more satisfied, compared with the control group, the difference was significant, $\mathrm{P}<0.05$. Further explanation, the use of psychological care, nursing complications predictable formula, can effectively reduce the patient's anxiety, improve the quality of nursing care, the effect is obvious, it is worth to use in clinical practice.

\section{References}

[1] Yang Jishi, Zhou Liulin. Journal of Practical Clinical Medicine, Vol. 1 (2010) No 511, p.178-179

[2] Fei Guohua, Zhang Jianzeng. Qilu Nursing, Vol. 1 (2010) No 312, p.257-258

[3] Yao Fulan. The Chinese Medicine Modern Distance Education, Vol. 8 (2010) No 21, p.67-68

[4] Dong Mei. Modern Medicine, Vol. 1 (2010) No16, p.110 